\title{
Experimental Vaccines against Chagas Disease: A Journey through History
}

\author{
Olivia Rodríguez-Morales, ${ }^{1}$ Víctor Monteón-Padilla, ${ }^{2}$ \\ Silvia C. Carrillo-Sánchez, ${ }^{1}$ Martha Rios-Castro, ${ }^{1}$ Mariana Martínez-Cruz, ${ }^{1}$ \\ Alejandro Carabarin-Lima, ${ }^{3}$ and Minerva Arce-Fonseca ${ }^{1}$ \\ ${ }^{1}$ Department of Molecular Biology, Instituto Nacional de Cardiología Ignacio Chávez, Juan Badiano No. 1, \\ Colonia Sección XVI, 14080 Delegación Tlalpan, DF, Mexico City, Mexico \\ ${ }^{2}$ Centro de Investigaciones Biomédicas, Universidad Autónoma de Campeche, Avenida Agustín Melgar No. 3, \\ 24030 Campeche, CAM, Mexico \\ ${ }^{3}$ Centro de Investigaciones en Ciencias Microbiológicas, Benemérita Universidad Autónoma de Puebla, \\ 14 Sur y Avenida San Claudio, Ciudad Universitaria, 72570 Puebla, PUE, Mexico
}

Correspondence should be addressed to Minerva Arce-Fonseca; mini_arce@yahoo.com.mx

Received 3 March 2015; Revised 21 April 2015; Accepted 27 April 2015

Academic Editor: Xiao-Feng Yang

Copyright (c) 2015 Olivia Rodríguez-Morales et al. This is an open access article distributed under the Creative Commons Attribution License, which permits unrestricted use, distribution, and reproduction in any medium, provided the original work is properly cited.

\begin{abstract}
Chagas disease, or American trypanosomiasis, which is caused by the protozoan parasite Trypanosoma cruzi, is primarily a vector disease endemic in 21 Latin American countries, including Mexico. Although many vector control programs have been implemented, T. cruzi has not been eradicated. The development of an anti-T. cruzi vaccine for prophylactic and therapeutic purposes may significantly contribute to the transmission control of Chagas disease. Immune protection against experimental infection with T. cruzi has been studied since the second decade of the last century, and many types of immunogens have been used subsequently, such as killed or attenuated parasites and new DNA vaccines. This primary prevention strategy appears feasible, effective, safe, and inexpensive, although problems remain. The objective of this review is to summarize the research efforts about the development of vaccines against Chagas disease worldwide. A thorough literature review was conducted by searching PubMed with the terms "Chagas disease" and "American trypanosomiasis" together with "vaccines" or "immunization". In addition, reports and journals not cited in PubMed were identified. Publications in English, Spanish, and Portuguese were reviewed.
\end{abstract}

\section{Introduction}

Chagas disease, or American trypanosomiasis, is caused by the protozoan parasite Trypanosoma cruzi. Chagas disease is primarily a vector disease endemic in 21 Latin American countries, where it has a strong economic impact because it primarily affects economically active people. Approximately 10 million people are infected, and $>25$ million people are at risk of infection in endemic countries [1].

After years or decades that infection is acquired, from $10 \%$ to $30 \%$ of infected people develop symptoms of chronic phase. The heart is the most commonly affected organ; symptoms include arrhythmias, cardiomyopathy, and thromboembolism. Death usually occurs from heart failure [2].
Although vector control and blood bank serological screening have greatly reduced parasite transmission, the costs to maintain such control programs as well as the differences between vector species, the existence of animal reservoirs, the parasite persistence in chronically infected people, and lack of adequate chemotherapy for the treatment of infection have made the complete eradication of T. cruzi impossible. An additional measure which could significantly contribute to the control of this disease is the development of an anti-T. cruzi vaccine [3-5]. The immunological protection against experimental infection with $T$. cruzi has been studied since the second decade of the last century and many types of immunogens such as killed or attenuated parasites and the newest DNA vaccines have been tested. 


\section{History}

Since 1912, when Blanchard demonstrated that animals surviving acute infection with $T$. cruzi became resistant to reinfection, active immunization against this protozoan began to be researched. Blanchard's experiments were confirmed by Brumpt, Mayer, and Rocha-Lima who used trypanosome blood forms for their studies [6].

In 1952, for the first time, Pizzi and Prager used cultured parasite attenuated forms to protect animals against infection with a T. cruzi virulent strain. Two years later, Rubio showed that infection with these cultured forms was exacerbated by corticosteroids. Various and partial results were obtained when live attenuated forms were tested in other immunization attempts of laboratory animals [7]. Immunization with dead trypanosomes began in 1912 with Laveran. Since then, several chemical and physical methods to kill parasites were evaluated, always with unsatisfactory results, except for those of Goble and colleagues who used a pressure chamber to dislodge the parasite and generate a vaccine [6].

González Cappa and colleagues observed that $88 \%$ and $100 \%$ of the animals, depending on the dosage and number of immunizations, were protected using antigens prepared in a pressure chamber [8].

In 1968, Menezes determined that the Y strain cultured for 15 years became avirulent, most likely because of mutations [9]. It was experimentally demonstrated that this strain protected animals against infection with different T. cruzi strains, suggesting that virulence factors are not essential as immunogens.

\section{Live, Killed, or Attenuated Parasite Immunization}

Live trypanosome immunization as well as killed or attenuated parasites for the preparation of immunogens that use physical or chemical methods has been performed. A brief overview of this type of immunization is presented below.

Epimastigotes of Trypanosoma rangeli fixed with glutaraldehyde and emulsified with saponin as an adjuvant were injected by laboratory of Introini. Mice were infected with 100 trypomastigotes of the Tulahuén T. cruzi strain [10]. The results showed that 2 or 3 doses are required to induce a significant reduction in parasitemia and increase survival.

Apart from T. rangeli, other trypanosomatids have been used as vaccines against T. cruzi. Breganó and colleagues immunized $\mathrm{BALB} / \mathrm{c}$ mice either intraperitoneally or orally with Phytomonas serpens, a tomato parasite that shares antigens with T. cruzi, and after lethal challenge with trypomastigotes, the mice showed a significant decrease in parasitemia and increased survival [11].

Inducible nitric oxide synthase (iNOS) knockout mice and C57BL/6 mice treated with an iNOS inhibitor were immunized orally with Phytomonas serpens and challenged with T. cruzi. A reduction in parasitemia and increased survival of C57BL/6 mice compared to the knockout animals were observed [12], suggesting that nitric oxide is a mechanism of parasite control.
Basso and colleagues vaccinated $\mathrm{BALB} / \mathrm{c}$ mice with live or fixed epimastigotes of two $T$. rangeli strains and lower levels of parasitemia and an increased survival rate were observed after T. cruzi Tulahuén strain infection. Histology revealed a moderate lymphocytic infiltrate [13]. This study demonstrated that the antigens involved in the protection induced by $T$. rangeli are expressed in different strains of this parasite, suggesting that it may be useful in the preparation of vaccines. Years later they immunized guinea pigs with epimastigotes of $T$. rangeli emulsified with saponin and subsequently challenged them with Tulahuén strain of T. cruzi trypomastigotes. These guinea pigs showed significantly lower parasitemia and a discrete lymphocytic infiltrate in the cardiac and skeletal muscles. In the chronic phase, the histology was normal. The control group exhibited nests of amastigotes and histopathological changes compatible with chagasic myocarditis, endocarditis, and pericarditis [14]. The immunoprotection by $T$. rangeli was shown and this identified new possible preventive tools that may reduce the risk of infection with T. cruzi.

In addition to attenuated parasites, animal models have also been immunized with mutant strains. The research group of Basombrio generated monoallelic mutant parasites for the $d h f r$-ts gene from a naturally attenuated strain. The mutant clones showed reduced virulence in mice. Moreover, there were fewer specific $\mathrm{CD}^{+} \mathrm{T}$ cells targeting T. cruzi. Mice challenged with virulent parasites one year after the original infection with the mutant strain showed significant control over the secondary infection [15]. This study indicates that it is possible to generate genetically attenuated parasites that confer protection against new infections by T. cruzi.

Four doses of live parasites (Sylvio X10/4) administered at 3-week intervals were evaluated. One to two months after the last dose, the number of $\mathrm{CD}^{+} \mathrm{T}$ cells producing IFN$\gamma$ and memory cells and the proliferative response of $\mathrm{T}$ cells increased in the spleen. However, the challenge induced an increase in the serum IgG1 levels and mixed Th1/Th2 cytokine production. Moreover, there were no significant changes in heart injuries or subpatent parasitemias [16]. In conclusion, this study may help identify the elements necessary for a successful therapeutic vaccine that reduces human cardiomyopathy in chronically infected patients.

\section{Cell Fraction Immunization}

To identify the more immunogenic portion of the parasite that induces a protective immune response, a variety of cell fractionation studies have been performed.

Ruiz and colleagues obtained subcellular fractions of the Tulahuén strain of $T$. cruzi epimastigotes that were used to immunize mice. Subsequently, the mice were challenged with 25 blood trypomastigotes. The animals immunized with the pellet and supernatant showed positive xenodiagnosis, myocarditis, and myositis similar to the control animals.

Immunization with the flagellar portion resulted in partial protection against myocarditis development, fewer animals with positive xenodiagnosis, and no electrocardiographic changes. Fifty percent of animals immunized with the pellet or the supernatant, which were not challenged, 
had electrocardiographic changes and myocarditis. Immunization with the flagellar fraction in the absence of infection induced lesions similar to those in the control animals [17]. These data are troubling because they demonstrate that the fractions alone can produce damage, possibly by inducing an autoimmune response.

Wrightsman and colleagues used the paraflagellar rod protein (PAR) purified from T. cruzi epimastigotes to protect mice against a lethal inoculum of $10^{3}$ blood trypomastigotes. Subcutaneous injection of the PAR proteins reduced parasitemia and showed $100 \%$ survival after challenge. By contrast, the intraperitoneal route induced parasitemia levels equivalent to the control animals and the mice did not survive the infection [18]. One year later, the PAR protein was administered subcutaneously in combination with Freund's adjuvant or aluminum and the survival was $100 \%$ and $83 \%$, respectively. The levels of IFN- $\gamma$ and IL-2 were higher in the protective groups, indicating that the protective immunity in mice immunized with PAR was associated with a Thl-type response [19]. The induction of an immune response associated with the mucous, serous tissue, or spleen is not ideal.

\section{Purified Protein Immunization}

Immunization studies with purified proteins from the parasite to identify those that induce an immune response and provide protection to the challenge have been performed.

Snary compared the ability of two T. cruzi surface glycoproteins to confer protection against experimental infection. The $90 \mathrm{kDa}$ glycoprotein found in all stages of T. cruzi protected against challenge with blood and metacyclic trypomastigotes. The $72 \mathrm{kDa}$ glycoprotein found only in stages derived from insects protected against challenge with metacyclic trypomastigotes only [20]. These data indicate that selection of the immunogenic protein is important and must be present in the parasite stages that circulate in the mammal.

A protective study using 45 and $68 \mathrm{kDa}$ antigens purified from the cell membrane of T. cruzi epimastigotes was performed by Araujo and Morein in 1991. The antigens were purified by affinity chromatography and incorporated into a system prepared with Quil A, a saponin derivative, for the immunization. A strong humoral and cellular response protected $100 \%$ of the immunized animals challenged with blood trypomastigotes [21].

Gomes and colleagues immunized C57B1/10 mice with an antigenic preparation called TcY 72, obtained when a lysate of T. cruzi Y strain epimastigotes was separated by electrophoresis and a $72 \mathrm{kDa}$ band was isolated by electroelution. They observed the induction of high levels of IgG antibodies, a delayed hypersensitivity reaction after injection of epimastigotes in the legs of the mice, a significant reduction of parasitemia, and a decreased CD4/CD8 ratio in immunized mice [22], which suggest a possible role for $\mathrm{CD}^{+}$subpopulations.

Taibi and his group investigated the immunoprotective properties of trypomastigotes excretory-secretory antigens. The ESA immunization of $\mathrm{BALB} / \mathrm{c}$ mice resulted in reduced parasitemia during acute infection and significant protection with $60 \%$ survival, whereas no mice in the control group survived after 39 days postinfection. The same experiments were performed in Fisher rats and showed greater protection against lethal infection with $100 \%$ survival [23].

The Gruppi group immunized mice with antigens released into the circulation, called exoantigens of pI 4.5, and observed partial protection. In subsequent experiments, they transferred the lymph node cells from immunized mice to normal recipients that were challenged, thereby reducing the parasitemia levels [24].

Garcia and colleagues administered T. cruzi soluble extract (TCSE) as an antigen in mice and analyzed the immune response when challenged with T. cruzi. The proliferative response in the animals upon stimulation with Concanavalin A was increased as was the IFN- $\gamma$ level. The production of IFN- $\gamma$ is important in controlling parasite replication because the protection by TCSE protecting was completely abrogated by in vivo treatment with a neutralizing anti-IFN- $\gamma$ [25]. These data suggest that despite the antigenic complexity, it is possible to generate similar protection using a macromolecule or a set of macromolecules.

\section{Recombinant Protein Immunization}

The modern biotechnology allows obtaining many copies of a parasite gene (producing an immunogenic protein) inserted into the bacterial DNA in a short time and producing large quantities of recombinant proteins.

Pereira and his group immunized BALB/c mice subcutaneously three times with recombinant $T$. cruzi cytoplasmic repetitive antigen (CRA) and flagellar repetitive antigen (FRA). The recombinant CRA produced high IgG3 and IgG1 levels, whereas only IgG1 was induced by recombinant FRA [26]. Pereira and his group suggested that the IgG3 isotype plays significant role in protection. The complexity or structure of the antigen may generate a different humoral response. In Chagas disease, the humoral immune response is important because antibodies can protect animals, thereby reducing parasitemia and mortality.

Luhrs et al. produced recombinant paraflagellar rod proteins (PFR), and mice immunized with these proteins against T. cruzi showed protection associated with a Th1 cytokine profile. These authors analyzed the PFR-2 gene sequence in seven highly diverse strains of $T$. cruzi and found that it is highly conserved. Immunization with PFR-1, PFR-2, PFR-3, or an equimolar mixture induced a $70 \%, 73 \%, 51 \%$, and $74 \%$ reduction in peak parasitemia, respectively. Of the animals immunized with PFR-3, $42 \%$ survived, whereas the remaining animals reached $100 \%$ survival [27]. It is important to identify new protective epitopes to optimize the design of vaccines.

Immunization of A/Sn mice with plasmid p154/13, which encodes a T. cruzi trans-sialidase, induced a Thl-type immune response. By contrast, immunization with the recombinant trans-sialidase generated a Th2-type immune response. Simultaneous administration of plasmid p154/13 and the recombinant protein in animals induced a Th2-type response, whereas when the first immunization was performed with two doses of plasmid followed by the recombinant protein, 
a Th1-type response was obtained as revealed by the reduction in the serum $\operatorname{IgG} 1 / \operatorname{IgG} 2 \mathrm{a}$ ratio and an increase of in vitro IFN- $\gamma$ produced by CD4 T cells [28]. This study shows that immunization with DNA followed by immunization with a recombinant protein may increase the Thl-type response in DNA immunization protocols. The mechanism underlying these results is unclear; however, double stranded plasmid DNA may interact with toll-like receptors, stimulating the production of IL-12, which in turn stimulates the development of a Thl-type response.

Cazorla et al. inoculated mice with the C-terminal domain of cruzipain and high titers of IgG2a were obtained. T. cruzi invasiveness is blocked by antibodies from mice immunized with recombinant cruzipain and with the $\mathrm{N}$-terminal domain. Mice immunized with the N-terminal domain and challenged with $T$. cruzi showed a lower concentration of enzymes associated with cardiomyopathy, such as creatine kinase and lactate dehydrogenase (LDH) [29]. This study shows that the N-terminal domain of cruzipain can produce a differential immune response, which may protect against a sublethal challenge with trypomastigotes.

Flores-García et al. evaluated the role of the T. cruzi recombinant protein $\mathrm{rMBP}: \mathrm{SSP} 4$ with a GPI anchor as an immunomodulatory molecule. They showed the secretion in vivo of several cytokines such as IL-4, IL-10, IFN- $\gamma$, and TNF- $\alpha$. The same cytokine profile was found in vitro, and besides IL-10 and IFN- $\gamma$ were particularly secreted by $\mathrm{CD} 4^{+}$ cells [30]. This cell population suppresses lymphocyte activity and plays an important role in regulating the immune response preventing a collateral damage generated by a strong immune response against the parasite, thus avoiding unwanted excessive tissue inflammation that would otherwise be exacerbated; this was proposed when mice were immunized with TcSP2-CHP and mice showed increased IL-2, IFN- $\alpha$, and IL-10 cytokines after parasite change and protection in heart was observed [31]. In addition, it is involved in the prolonged persistence of the parasite and protection against severe inflammatory responses in the host.

A new antigen-adjuvant combination for protection against experimental Chagas disease was assessed. The antigen used in the formulation was a glycosylated mutant inactive trans-sialidase (mTS) and the adjuvant used was ISCOMATRIX (IMX). The mice immunized with mTS-IMX showed a TS-specific IgG response, increased IgG2a/IgG1 ratio, significant delayed-type hypersensitivity (DTH) reactivity, balanced production of IFN- $\gamma$ and IL-10 by splenocytes, and strong IFN- $\gamma$ secretion by $\mathrm{CD}^{+}{ }^{+} \mathrm{T}$ lymphocytes. All mTS-IMX immunized infected mice showed $\sim 50$ times less parasitemia than nonimmunized infected mice; in the chronic phase, tissue presented $\sim 4.5$ times lower parasite load. These results indicate that mTS-IMX formulation induces both optimal humoral and cellular immune responses, conferring protection against T. cruzi [32].

\section{DNA Vaccines against Chagas Disease}

DNA vaccines provide an alternative for both prevention and treatment of a variety of infectious diseases [33-37], including
Chagas disease. TcSP gene (encoding a member of the transsialidase superfamily), TcSSP4 gene (encoding an amastigotespecific surface protein), or their recombinant proteins were used in an immunization protocol in murine model challenging with the $\mathrm{H} 8$ strain of T. cruzi. Immunization with the rTcSP recombinant protein or the $T c S P$ gene gave a mixed Th1/Th2 $\mathrm{T}$ cell immune response. The mice vaccinated with TcSSP 4 showed significant amounts of IFN- $\gamma$, suggesting a Th1 response. Only the mice immunized with DNA showed a significant reduction in the parasitemia peak and lethal challenge survival $[38,39]$. These studies demonstrate that DNA immunization induces a protective immune response in contrast to the use of homologous recombinant protein in the experimental infection with T. cruzi.

The prophylactic use of these two genes in Beagle dogs with Chagas disease showed that the dominant antibodies were subclass IgG2 immunoglobulins. It was also demonstrated that both genes' immunization induced cell mediated immunity characterized by lymphoproliferation as well as IFN- $\gamma$ production [40]. Immunization decreased the quality and quantity of electrocardiographic abnormalities, thereby avoiding progression to more severe cardiac disturbances [41]. A partial protective effect for the prevention of macroscopic and microscopic damage in cardiac tissue during the chronic phase was also observed in these Beagle dogs [42]. These two genes of T. cruzi that generate a moderate level of protection in the chronic phase of the disease were proposed as vaccine candidates.

The immune response elicited by TcVac3 (antigenic candidates TcG2 and TcG4, membrane-associated GPI proteins) delivered by a DNA-prime/MVA-boost approach against challenge to the parasite in C57BL/6 mice has been characterized. The vaccine elicited a strong antigen- and parasitespecific, high avidity, lytic, Th1-type antibody response; it also elicited antigen- and parasite-specific CD4/CD8 T cell proliferation. The $\mathrm{CD}^{+} \mathrm{T}$ cells were predominantly IFN $-\gamma^{+}$ with cytolytic capacity. In chronic phase, immunized mice exhibited a massive decline in proinflammatory phenotype, a predominance of immunoregulatory IL- $10^{+} / \mathrm{CD} 4^{+} \mathrm{T}$ and IL- $10^{+} / \mathrm{CD}^{+} \mathrm{T}$ cells, and presented undetectable tissue parasitism, inflammatory infiltrate, and fibrosis [43]. In this study, the emergence of type 2 cytokine and $\mathrm{T}$ cell response suggested prevention of clinical disease.

The backbone of Yellow Fever (YF) 17D virus to express an immunogenic fragment derived from T. cruzi Amastigote Surface Protein-2 (ASP-2) was used. A/J mice were immunized subcutaneously with two doses of recombinant virus and four weeks after the last dose they were challenged with T. cruzi. Immunogenicity studies showed a reduction in mouse mortality, an increase of average survival time, and a reduction in peak parasitemia. The amount of IFN- $\gamma$ secreting splenocytes significantly increased for all mice immunized compared to the negative control group [44]. The results suggested expanding the applicability of YF 17D as a potential recombinant virus vector to express other antigens for Chagas disease vaccine development.

A DNA vaccine encoding TSA- 1 and Tc24 antigens in a dog model of acute T. cruzi infection was evaluated. Mongrel dogs were immunized with two doses of $500 \mu \mathrm{g}$ of DNA 
vaccine and infected with SylvioX10/4 strain of T. cruzi two weeks after the second vaccine dose. Another group of dogs was infected first and treated with the vaccine. Both preventive and therapeutic vaccination significantly reduced parasitemia, cardiac inflammation, and cardiac parasite burden and tended to reduce the development of cardiac arrhythmias. The effect of vaccination on the immune response was weak because it did not observe the induction of a humoral response; however, an increased level of IFN$\gamma$ in immunized dogs was detected, suggesting that DNA vaccination tended to prime a cellular immune response [45]. These results demonstrated that the use of antigens expressed on DNA plasmids may be successful as preventive and therapeutic veterinary vaccine candidates in reducing both parasite transmission and the clinical progression of Chagas disease in vaccinated dogs and possibly other pets.

The TcG1, TcG2, and TcG4 antigens were delivered as a DNA prime/protein boost vaccine (TcVac2) in immunized C57BL/6 mice. Vaccinated mice showed lytic antibodies and type $1 \mathrm{CD}^{+} \mathrm{T}$ cells that expanded upon challenge infection and provided $>90 \%$ control of parasite burden and myocarditis in chagasic mice. Macrophages incubated with sera from vaccinated infected mice exhibited M2 surface markers (CD16, CD32, CD200, and CD206), moderate proliferation, a low oxidative/nitrosative burst as an indicator of low phagocyte cytotoxicity, and a regulatory/anti-inflammatory cytokine response (interleukin-4 [IL-4] plus IL-10 > tumor necrosis factor alpha [TNF- $\alpha]$ ] [46]. The results suggested that the TcVac2 vaccine controls chronic myocarditis due to the antiproliferative and anti-inflammatory responses of macrophages. These findings open the possibility of using the in vitro phenotypic and functional profiling of macrophages to assess the efficacy of prophylactic and therapeutic vaccine candidates.

Vasconcelos et al. employed a heterologous prime-boost vaccination using plasmid DNA followed by replicationdefective adenovirus vector (expressing the ASP-2 gene) in the gzmBCreERT2/ROSA26EYFP transgenic mouse line. They observed that the frequency of the specific $\mathrm{CD} 8^{+} \mathrm{T}$ cells in the spleens of the vaccinated mice increased after challenge and the increase in the frequency of specific cells and the protective immunity they mediate were insensitive to treatment with the cytostatic toxic agent hydroxyurea. Specific $\mathrm{CD}^{+} \mathrm{T}$ cells were capable of producing simultaneously the antiparasitic mediators IFN- $\gamma$ and TNF [47]. This group suggested that differentiation and recirculation, rather than proliferation, are key for the resultant protective immunity.

Cazorla et al. determined whether immunoprotection elicited by the cysteine protease cruzipain $(\mathrm{Cz})$ could be improved by coadministration of Salmonella strains carrying plasmids encoding a thiol-transferase (Tc52) and a $24 \mathrm{kDa}$ flagellar calcium-binding protein (FCaBP or Tc24) of $T$. cruzi. Immunized $\mathrm{C} 3 \mathrm{H} / \mathrm{HeN}$ mice with a multicomponent oral vaccine elicited strong humoral and cellular immune responses, conferring improved protection against T. cruzi infection with respect to monocomponent formulations [48]. This study demonstrates that the approach of protective effect searching the combination of several antigens involved in invasion or metabolic processes may highlight the benefits of a better immune response than vaccines with a single antigen.

The exploration of human vaccines against $T$. cruzi has been widely avoided due to the fear that this prophylactic measure could exacerbate the disease that many still consider to have an autoimmune etiology, although the evidence suggests that the best correlation between the induction and maintenance of inflammatory disease process is the persistence of the parasite in tissues of patients and not the immune response induced by the parasite [49].

The lack of financial support from governments and the pharmaceutical industry has been another factor that has greatly contributed to the disinterest in this field due to the fact that American trypanosomiasis is one of the diseases that affected the poorest people living in the Americas.

Chagas disease is included in the group of chronic parasitic, bacterial, and viral infections that actually promote poverty in people because they affect child development, pregnancy outcome, and worker productivity, so it is considered a Neglected Tropical Disease (NTD) [50].

Currently a therapeutic Chagas vaccine is under development by a consortium of Mexican (including the Carlos Slim Health Institute) and Texan scientific institutions; based on the evidence of therapeutic efficacy of Tc24 and TSA1 vaccines in reducing $T$. cruzi-induced cardiac disease in mice and dogs, the authors are pursuing Tc24 and TSA- 1 as lead candidate antigens. These researchers argued in 2012 that the two antigens comprising a prototype therapeutic vaccine for human Chagas disease could be produced under current Good Manufacturing Practices within the next five years [51].

\section{Conclusions}

The development of an effective human vaccine against Chagas disease has encountered many difficulties, and progress has been slow mainly because there is still controversy about its autoimmune etiology and disinterest of the authorities in endemic countries where this NTD exists. The genetic complexity of T. cruzi as well as the limited set of efficient engineering techniques for genome manipulating contributes significantly to the relative lack of progress in the understanding of this microorganism.

Despite several T. cruzi proteomic studies, the evaluation of bloodstream trypomastigotes profile remains unexplored. The approach of proteomic studies to develop vaccines that include antigens from both intracellular (amastigote) and extracellular (trypomastigote) forms is necessary.

The use of defined native antigens purified from T. cruzi is convenient and acceptable, but it is difficult to obtain these antigens in the volumes necessary to perform the studies. Monoclonal antibody production and advances in the methods for obtaining proteins have improved the identification, isolation, and purification of defined parasite proteins but increase the difficulty in achieving sufficient and sustainable vaccine production.

Obtaining antigens using molecular biology techniques and recombinant DNA have enabled the cloning, expressing, and production of T. cruzi antigens. These available molecular 
tools, combined with the knowledge of several parasite antigens, have allowed the development of new DNA vaccines. Furthermore, to enhance the immune response generated by DNA immunization with genes encoding molecules that stimulate the immune system, they have been coadministered with some T. cruzi genes.

Because there is no effective treatment for patients in acute stage, DNA could control T. cruzi infection and significantly reduce the progression of chronic Chagasic cardiomyopathy. The effectiveness of this type of vaccination is greater than conventional vaccine because DNA vaccines induce IFN- $\gamma$ secretion and stimulate Thl helper T cells, which are necessary to confer protection against infection with this parasite. However, it is important when selecting a vaccine containing genes or fragments thereof to demonstrate that the gene is present in the majority of T. cruzi strains, and if peptides are used, it must be demonstrated that the population immunized with this peptide expresses the correct MHC for presentation to CD4 or CD8 lymphocytes, as applicable. Besides, in chronically infected patients, it would be important to develop a therapeutic vaccine to induce the parasite complete elimination by the immune system.

The ideal prophylactic vaccination would be one that does not allow the parasite establishment or reduce the parasite burden, so it has a scientific basis for the use of immunization in humans and domestic reservoirs in endemic areas for prevention and control of Chagas disease.

\section{Conflict of Interests}

The authors declare that there is no conflict of interests regarding the publication of this paper.

\section{References}

[1] World Health Organization, "Weekly epidemiological record," vol. 87, no. 51-52, pp. 509-526, 2012, http://www.who.int/wer.

[2] L. V. Kirchhoff, "American trypanosomiasis (Chagas' disease) a tropical disease now in the United States," The New England Journal of Medicine, vol. 329, no. 9, pp. 639-644, 1993.

[3] B. Wizel, N. Garg, and R. L. Tarleton, "Vaccination with trypomastigote surface antigen 1-encoding plasmid DNA confers protection against lethal Trypanosoma cruzi infection," Infection and Immunity, vol. 66, no. 11, pp. 5073-5081, 1998.

[4] R. L. Tarleton and J. W. Curran, "Is Chagas disease really the 'new HIV/AIDS of the Americas'?" PLoS Neglected Tropical Diseases, vol. 6, no. 10, Article ID e1861, 2012.

[5] K. J. Esch and C. A. Petersen, "Transmission and epidemiology of zoonotic protozoal diseases of companion animals," Clinical Microbiology Reviews, vol. 26, no. 1, pp. 58-85, 2013.

[6] A. Romero-Davalos, Enfermedad de Chagas, Editorial Los Amigos del Libro, La Paz, Bolivia, 1979.

[7] T. Pizzi and R. Prager, "Immunity to infection induced by culture of Trypanosoma cruzi of atenuated virulence; preliminary communication," Boletín Informativo de Parasitolgía Chilena, vol. 7, no. 2, pp. 20-21, 1952.

[8] S. M. González Cappa, G. A. Schmuñis, O. C. Traversa, J. F. Yanovsky, and A. S. Parodi, "Complement-fixation tests, skin tests, and experimental immunization with antigens of
Trypanosoma cruzi prepared under pressure," The American Journal of Tropical Medicine and Hygiene, vol. 17, no. 5, pp. 709715, 1968.

[9] H. Menezes, "Protective effect of an avirulent (cultivated) strain of Trypanosoma cruzi against experimental infection in mice," Revista do Instituto de Medicina Tropical de Sao Paulo, vol. 10, no. 1, pp. 1-4, 1968.

[10] M. V. Introini, B. Basso, and E. Moretti, "Experimental Chagas' disease: I. Study of different immunization conditions in the infection course," Boletín chileno de parasitología, vol. 53, no. 34, pp. 45-51, 1998.

[11] J. W. Breganó, R. C. Picão, V. K. Graça et al., "Phytomonas serpens, a tomato parasite, shares antigens with Trypanosoma cruzi that are recognized by human sera and induce protective immunity in mice," FEMS Immunology \& Medical Microbiology, vol. 39, no. 3, pp. 257-264, 2003.

[12] P. Pinge-Filho, J. P. S. Peron, T. R. de Moura et al., "Protective immunity against Trypanosoma cruzi provided by oral immunization with Phytomonas serpens: role of nitric oxide," Immunology Letters, vol. 96, no. 2, pp. 283-290, 2005.

[13] B. Basso, E. Moretti, and R. Fretes, "Vaccination with epimastigotes of different strains of Trypanosoma rangeli protects mice against Trypanosoma cruzi infection," Memórias do Instituto Oswaldo Cruz, vol. 103, no. 4, pp. 370-374, 2008.

[14] B. Basso, E. Moretti, and R. Fretes, "Vaccination with Trypanosoma rangeli induces resistance of guinea pigs to virulent Trypanosoma cruzi," Veterinary Immunology and Immunopathology, vol. 157, no. 1-2, pp. 119-123, 2014.

[15] C. Perez Brandan, A. M. Padilla, D. Xu, R. L. Tarleton, and M. A. Basombrio, "Knockout of the dhfr-ts gene in Trypanosoma cruzi generates attenuated parasites able to confer protection against a virulent challenge," PLoS Neglected Tropical Diseases, vol. 5, no. 12, article e1418, 2011.

[16] C. E. Rosas-Jorquera, L. R. Sardinha, F. D. Pretel, A. L. Bombeiro, M. R. D. Lima, and J. M. Alvarez, "Challenge of chronically infected mice with homologous Trypanosoma cruzi parasites enhances the immune response but does not modify cardiopathy: implications for the design of a therapeutic vaccine," Clinical and Vaccine Immunology, vol. 20, no. 2, pp. 248254, 2013.

[17] A. M. Ruiz, M. Esteva, P. Cabeza Meckert, R. P. Laguens, and E. L. Segura, "Protective immunity and pathology induced by inoculation of mice with different subcellular fractions of Trypanosoma cruzi," Acta Tropica, vol. 42, no. 4, pp. 299-309, 1985.

[18] R. A. Wrightsman, M. J. Miller, J. L. Saborio, and J. E. Manning, "Pure paraflagellar rod protein protects mice against Trypanosoma cruzi infection," Infection and Immunity, vol. 63, no. 1, pp. 122-125, 1995.

[19] M. J. Miller, R. A. Wrightsman, and J. E. Manning, "Trypanosoma cruzi: protective immunity in mice immunized with paraflagellar rod proteins is associated with a T-helper type 1 response," Experimental Parasitology, vol. 84, no. 2, pp. 156-167, 1996.

[20] D. Snary, "Cell surface glycoproteins of Trypanosoma cruzi: protective immunity in mice and antibody levels in human chagasic sera," Transactions of the Royal Society of Tropical Medicine and Hygiene, vol. 77, no. 1, pp. 126-129, 1983.

[21] F. G. Araujo and B. Morein, "Immunization with Trypanosoma cruzi epimastigote antigens incorporated into iscoms protects against letal challenge in mice," Infection and Immunity, vol. 59, no. 9, pp. 2909-2914, 1991. 
[22] Y. M. Gomes, F. G. C. Abath, M. Nakazawa, P. Minoprio, I. Vouldoukis, and L. Monjour, "Partial protection of mice against Trypanosoma cruzi after immunizing with the TcY 72 antigenic preparation," Memórias do Instituto Oswaldo Cruz, vol. 94, no. 2, pp. 167-172, 1999.

[23] A. Taibi, B. Plumas-Marty, A. Guevara-Espinoza et al., "Trypanosoma cruzi: immunity-induced in mice and rats by trypomastigote excretory-secretory antigens and identification of a peptide sequence containing a $\mathrm{T}$ cell epitope with protective activity," The Journal of Immunology, vol. 151, no. 5, pp. 26762689, 1993.

[24] A. Gruppi, F. Cerban, M. C. Pistoresi-Palencia, and E. VotteroCima, "Trypanosoma cruzi: transfer of protection by lymph node cells obtained from mice immunized with exoantigens of pI 4.5," Experimental Parasitology, vol. 80, no. 3, pp. 382-389, 1995.

[25] C. A. A. C. Garcia, E. C. Oliveira, J. K. Sakurada, and L. M. B. Santos, "Protective immunity induced by a Trypanosoma cruzi soluble extract antigen in experimental Chagas' disease. Role of interferon $\gamma$," Immunological Investigations, vol. 29, no. 1, pp. 1$12,2000$.

[26] V. R. A. Pereira, V. M. B. Lorena, A. F. A. Verçosa et al., "Antibody isotype responses in $\mathrm{BALB} / \mathrm{c}$ mice immunized with the cytoplasmic repetitive antigen and flagellar repetitive antigen of Trypanosoma cruzi," Memorias do Instituto Oswaldo Cruz, vol. 98, no. 6, pp. 823-825, 2003.

[27] K. A. Luhrs, D. L. Fouts, and J. E. Manning, "Immunization with recombinant paraflagellar rod protein induces protective immunity against Trypanosoma cruzi infection," Vaccine, vol. 21, no. 21-22, pp. 3058-3069, 2003.

[28] J. R. C. Vasconcelos, S. B. Boscardin, M. I. Hiyane, S. S. Kinoshita, A. E. Fujimura, and M. M. Rodrigues, "A DNApriming protein-boosting regimen significantly improves type 1 immune response but not protective immunity to Trypanosoma cruzi infection in a highly susceptible mouse strain," Immunology and Cell Biology, vol. 81, no. 2, pp. 121-129, 2003.

[29] S. I. Cazorla, F. M. Frank, P. D. Becker et al., "Redirection of the immune response to the functional catalytic domain of the cystein proteinase cruzipain improves protective immunity against Trypanosoma cruzi infection," The Journal of Infectious Diseases, vol. 202, no. 1, pp. 136-144, 2010.

[30] Y. Flores-García, J. L. Rosales-Encina, A. R. Satoskar, and P. Talamás-Rohana, "IL-10-IFN- $\gamma$ double producers CD4+ T cells are induced by immunization with an amastigote stage specific derived recombinant protein of Trypanosoma cruzi," International Journal of Biological Sciences, vol. 7, no. 8, pp. 10931100, 2011.

[31] A. Carabarin-Lima, M. C. González-Vázquez, L. BaylonPacheco, V. Tsutsumi, P. Talamás-Rohana, and J. L. RosalesEncina, "Immunization with the recombinant surface protein rTcSP2 alone or fused to the CHP or ATPase domain of TcHSP70 induces protection against acute Trypanosoma cruzi infection," Journal of Vaccines \& Vaccination, vol. 1, no. 3, 2011.

[32] I. A. Bontempi, M. H. Vicco, G. Cabrera et al., "Efficacy of a trans-sialidase-ISCOMATRIX subunit vaccine candidate to protect against experimental Chagas disease," Vaccine, vol. 33, no. 10, pp. 1274-1283, 2015.

[33] J. L. Dutton, B. Li, W.-P. Woo et al., "A novel DNA vaccine technology conveying protection against a lethal herpes simplex viral challenge in mice," PLoS ONE, vol. 8, no. 10, Article ID e76407, 2013.
[34] G. Lu, A. Zhou, M. Meng et al., "Alpha-galactosylceramide enhances protective immunity induced by DNA vaccine of the SAG5D gene of Toxoplasma gondii," BMC Infectious Diseases, vol. 14, no. 1, p. 3862, 2014.

[35] S. Qiu, X. Ren, Y. Ben et al., "Fusion-expressed CTB improves both systemic and mucosal T-cell responses elicited by an intranasal DNA priming/intramuscular recombinant vaccinia boosting regimen," Journal of Immunology Research, vol. 2014, Article ID 308732, 6 pages, 2014.

[36] B. Dong, D. S. Zarlenga, and X. Ren, "An overview of live attenuated recombinant pseudorabies viruses for use as novel vaccines," Journal of Immunology Research, vol. 2014, Article ID 824630, 10 pages, 2014.

[37] Y. Xu, N. Z. Zhang, Q. D. Tan et al., "Evaluation of immunoefficacy of a novel DNA vaccine encoding Toxoplasma gondii rhoptry protein 38 (TgROP38) against chronic toxoplasmosis in a murine model," BMC Infectious Diseases, vol. 14, no. 1, article 525, 2014.

[38] B. Salgado-Jiménez, M. Arce-Fonseca, L. Baylón-Pacheco, P. Talamás-Rohana, and J. L. Rosales-Encina, "Differential immune response in mice immunized with the $\mathrm{A}, \mathrm{R}$ or $\mathrm{C}$ domain from TcSP protein of Trypanosoma cruzi or with the coding DNAs," Parasite Immunology, vol. 35, no. 1, pp. 32-41, 2013.

[39] M. Arce-Fonseca, A. Ramos-Ligonio, A. López-Monteón, B. Salgado- Jiménez, P. Talamás-Rohana, and J. L. Rosales-Encina, "A DNA vaccine encoding for TcSSP4 induces protection against acute and chronic infection in experimental Chagas disease," International Journal of Biological Sciences, vol. 7, no. 9, pp. 1230-1238, 2011.

[40] M. Arce-Fonseca, M. A. Ballinas-Verdugo, E. R. A. Zenteno et al., "Specific humoral and cellular immunity induced by Trypanosoma cruzi DNA immunization in a canine model," Veterinary Research, vol. 44, no. 1, article 15, 2013.

[41] O. Rodríguez-Morales, M. M. Pérez-Leyva, M. A. BallinasVerdugo et al., "Plasmid DNA immunization with Trypanosoma cruzi genes induces cardiac and clinical protection against Chagas disease in the canine model," Veterinary Research, vol. 43, no. 1, article 79, 2012.

[42] O. Rodríguez-Morales, S. C. Carrillo-Sánchez, H. GarcíaMendoza et al., "Effect of the plasmid-DNA vaccination on macroscopic and microscopic damage caused by the experimental chronic Trypanosoma cruzi infection in the canine Model," BioMed Research International, vol. 2013, Article ID 826570, 8 pages, 2013.

[43] S. Gupta and N. J. Garg, "TcVac3 induced control of Trypanosoma cruzi infection and chronic myocarditis in mice," PLoS ONE, vol. 8, no. 3, Article ID e59434, 2013.

[44] R. T. Nogueira, A. R. Nogueira, M. C. S. Pereira et al., "Recombinant yellow fever viruses elicit CD8+ T cell responses and protective immunity against Trypanosoma cruzi," PLoS ONE, vol. 8, no. 3, Article ID e59347, 2013.

[45] I. A. Quijano-Hernández, A. Castro-Barcena, J. C. VázquezChagoyán, M. E. Bolio-González, J. Ortega-López, and E. Dumonteil, "Preventive and therapeutic DNA vaccination partially protect dogs against an infectious challenge with Trypanosoma cruzi," Vaccine, vol. 31, no. 18, pp. 2246-2252, 2013.

[46] S. Gupta, T. S. Silva, J. E. Osizugbo, L. Tucker, H. M. Spratt, and N. J. Garg, "Serum-mediated activation of macrophages reflects TcVac2 vaccine efficacy against chagas disease," Infection and Immunity, vol. 82, no. 4, pp. 1382-1389, 2014. 
[47] J. R. Vasconcelos, M. R. Dominguez, R. L. Neves et al., "Adenovirus vector-induced $\mathrm{CD}^{+} \mathrm{T}$ effector memory cell differentiation and recirculation, but not proliferation, are important for protective immunity against experimental Trypanosoma cruzi infection," Human Gene Therapy, vol. 25, no. 4, pp. 350-363, 2014.

[48] S. I. Cazorla, M. N. Matos, N. Cerny et al., "Oral multicomponent DNA vaccine delivered by attenuated salmonella elicited immunoprotection against American trypanosomiasis," The Journal of Infectious Diseases, vol. 211, no. 5, pp. 698-707, 2015.

[49] S. I. Cazorla, F. M. Frank, and E. L. Malchiodi, "Vaccination approaches against Trypanosoma cruzi infection," Expert Review of Vaccines, vol. 8, no. 7, pp. 921-935, 2009.

[50] P. J. Hotez, A. Fenwick, L. Savioli, and D. H. Molyneux, "Rescuing the bottom billion through control of neglected tropical diseases," The Lancet, vol. 373, no. 9674, pp. 1570-1575, 2009.

[51] E. Dumonteil, M. E. Bottazzi, B. Zhan et al., "Accelerating the development of a therapeutic vaccine for human Chagas disease: rationale and prospects," Expert Review of Vaccines, vol. 11, no. 9, pp. 1043-1055, 2012. 


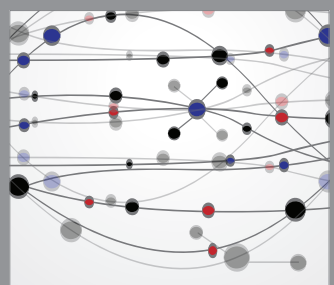

The Scientific World Journal
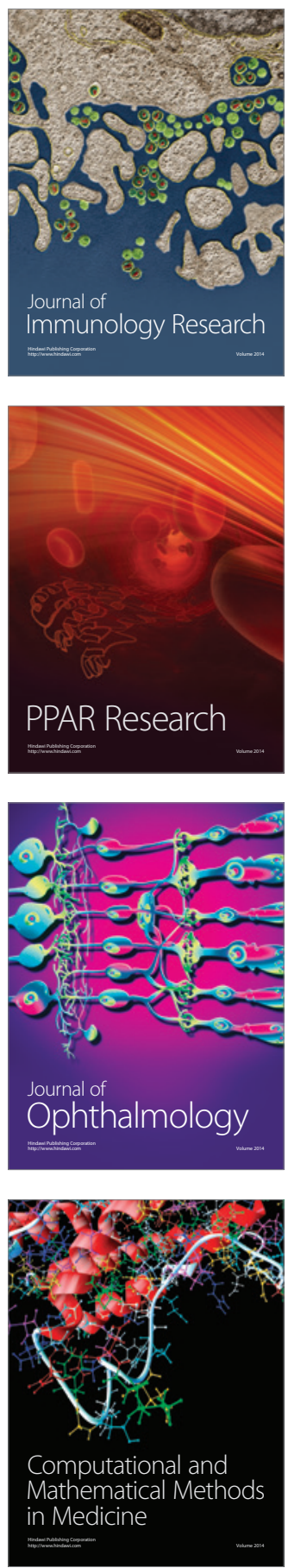

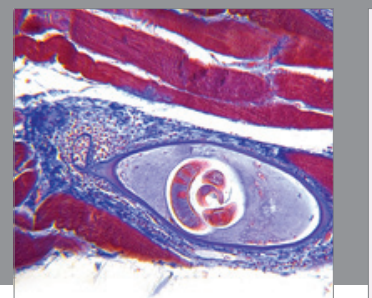

Gastroenterology

Research and Practice
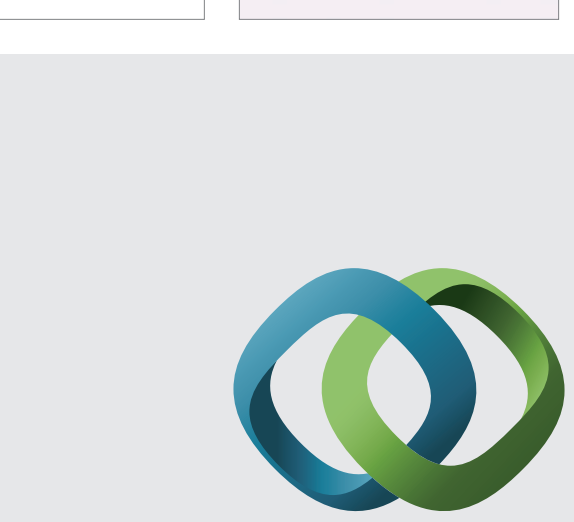

\section{Hindawi}

Submit your manuscripts at

http://www.hindawi.com
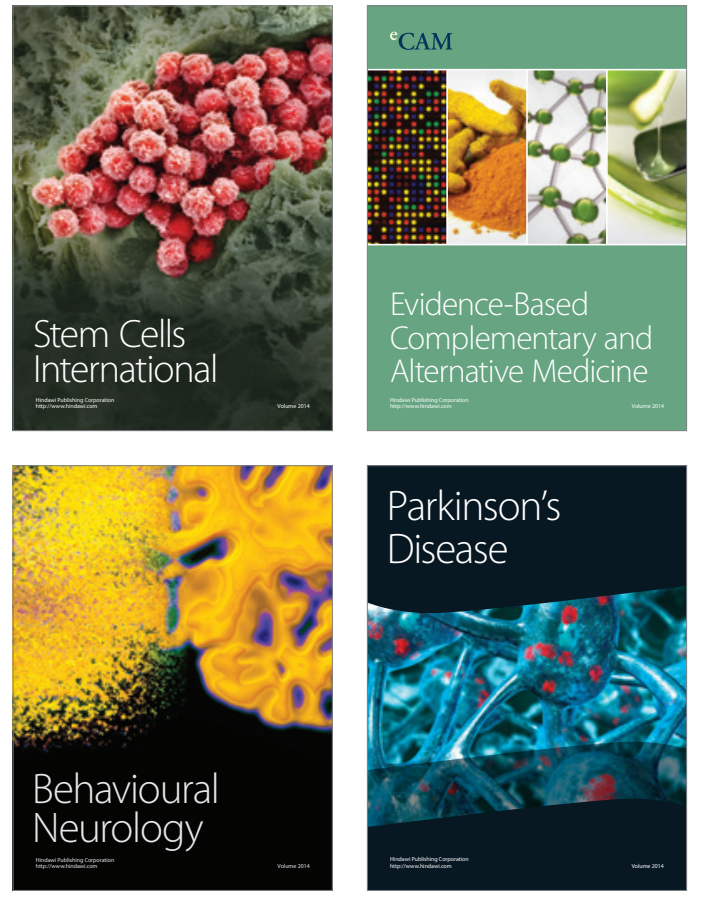
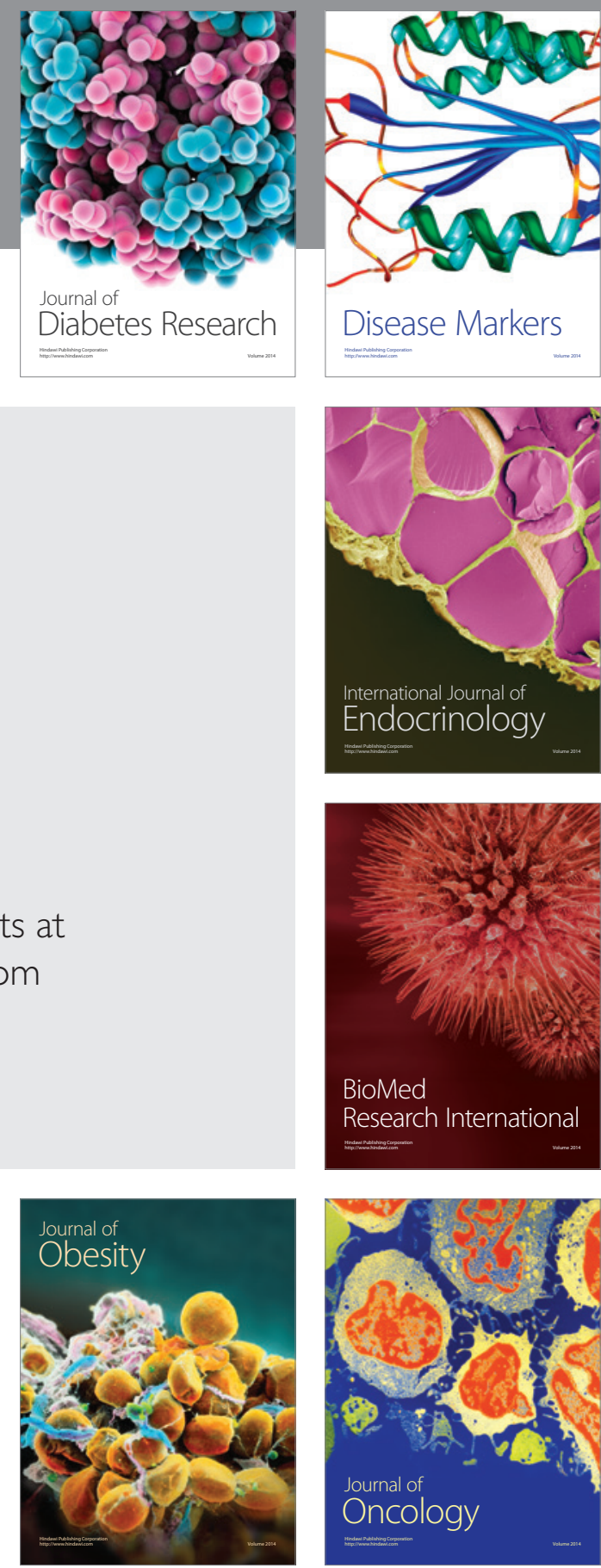

Disease Markers
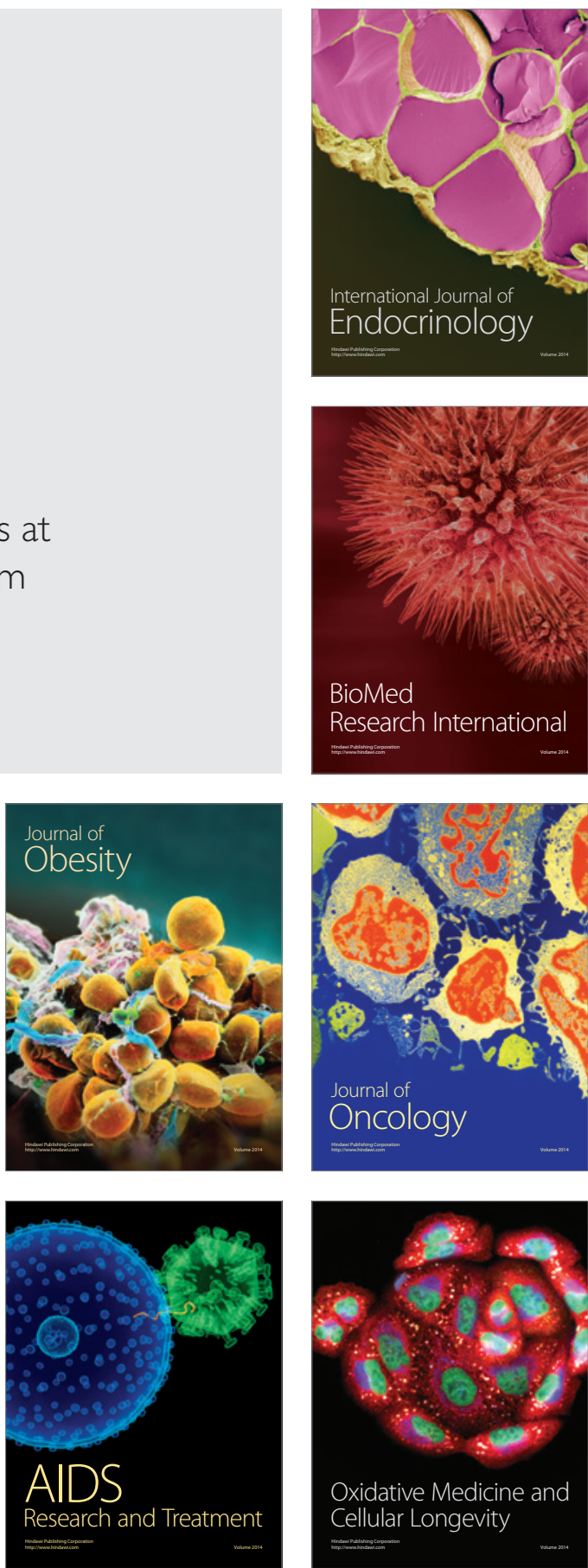